\title{
A Practical Scoring System to Select Optimally Sized Devices for Percutaneous Patent Foramen Ovale Closure
}

\author{
Joseph M. Venturini, MD*, Elizabeth M. Retzer, MD, J. Raider Estrada, MD, Anuj Mediratta, MD, \\ Janet Friant, MSN, Sandeep Nathan, MD, Jonathan D. Paul, MD, John Blair, MD, \\ Roberto M. Lang, MD, Atman P. Shah, MD
}

Section of Cardiology, Department of Medicine, University of Chicago, Chicago, Illinois, USA

\begin{abstract}
Background: Patent foramen ovale (PFO) has been linked to cryptogenic stroke, and closure has been reported to improve clinical outcomes. However, there are no clear guidelines to direct device sizing. This study sought to use patient characteristics and echocardiographic findings to create a prediction score for device sizing.

Methods: This was a retrospective review of patients undergoing percutaneous PFO closure at our institution between July 2010 and December 2014. Demographic and clinical characteristics were recorded, and all pre- and intraprocedural echocardiography results were evaluated. Results: Thirty-six patients underwent percutaneous PFO closure during the study period. All procedures were performed using an Amplatzer Septal Occluder "Cribriform" (ASOC) device in one of three disc diameters: $\mathbf{2 5}, \mathbf{3 0}$, or $35 \mathrm{~mm}$. Closure was indicated for cryptogenic stroke/transient ischemic attack in $75 \%$ of cases. Every case (100\%) was successful with durable shunt correction at the 6-month follow-up without complications of erosion or device embolization. The presence of atrial septal aneurysm (ASA) $(p=0.027)$ and PFO tunnel length $>10 \mathrm{~mm}(p=0.038)$ were independently associated with increased device size. A scoring system of 1 point for male sex, 1 point for ASA, and 1 point for PFO tunnel $>10 \mathrm{~mm}$ long was associated with the size of closure device implanted $(p=0.006)$.

Conclusions: A simple scoring system may be used to select an optimally sized device for percutaneous PFO closure using the ASOC device.

(c) 2016 Journal of Structural Heart Disease Published by Science International Corp. ISSN 2326-4004
Key Words

Patent foramen ovale - Device sizing - Percutaneous closure

\section{Introduction}

A patent foramen ovale (PFO) is a remnant of fetal circulation. In utero, placental blood from the inferior vena cava is directed toward the interatrial septum and across the foramen ovale, facilitating flow of oxygenated blood into systemic circulation. At birth, decreased pulmonary artery and right heart pressures result in a reversal of the right atrium-to-left atrium pressure gradient across the foramen ovale. This change in pressure pushes the septum primum (left atrial flap) against the septum secundum (muscular atrial septum). These structures typically fuse together in the first two years of life. Incomplete fusion results in a slit-like defect that is present in approximately $25 \%$ of adults and is referred to as a PFO [1,2]. The presence of PFO has been associated with an increased risk for paradoxical embolism resulting in stroke or obstructing peripheral embolism [3-7]. PFO has also been linked to increased risk for migraine headaches, hypoxemia, decompression syndrome in divers, and the platypnea-orthodeoxia syndrome [8-10]. The severity of these associated illnesses, particularly the potentially devastating complications of cryptogenic stroke, has increased interest in PFO closure.

\section{* Corresponding Author:}

Atman P. Shah, MD

University of Chicago Medicine

5841 S. Maryland Ave, M C6080, Chicago, IL 60637, USA

Tel.: +1 773702 1372; Fax: +1 773702 0241; E-Mail: ashah@bsd.uchicago.edu 
Transcatheter PFO closure may be performed with a variety of closure devices, which are manufactured in multiple sizes. Appropriate sizing of the selected device is critical to ensure adequate closure of the defect, minimize the likelihood of embolization, and avoid erosion of nearby cardiac structures. Too-large devices have been associated with erosion and an increased risk of atrial fibrillation [11, 12]. Conversely, devices that are too small increase the risk of incomplete closure and are at risk for embolization [13].

While there are clear guidelines for the sizing of atrial septal defect (ASD) closure devices, including use of the stop-flow technique, at this time, there is no consensus regarding the selection of closure device size for PFO closure when utilizing the Amplatzer Septal Occluder "Cribriform" (ASOC) device (St. Jude Medical, SJM; St. Paul, MN, USA) for off-label closure of symptomatic PFOs. The instructions for the use of the ASOC, which is intended for ASD closure, suggest device size selection that maximizes device size such that the radius of the discs does not exceed the shortest distance from the defect in the septum to either the aortic root or superior vena cava (SVC) [14]. These criteria ensure that the device is large enough to cover fenestrated ASDs without impinging surrounding structures. Sizing balloons may be used to approximate the diameter of a PFO [15]. However, the compliant nature of the septum primum and secundum make this technique imprecise, and the use of a sizing balloon carries the risk of interatrial septum rupture [16]. Currently, PFO device size is selected at the discretion of experienced operators, who may rely on anatomic factors (e.g., presence of interatrial septal aneurysm, tunnel length, shunt visualization) and clinical factors (e.g., patient age, sex, and body mass index (BMI)).

This study sought to identify patient characteristics and echocardiographic findings associated with size selection of the ASOC for PFO closure.

\section{Methods}

A retrospective review was performed of patients undergoing percutaneous PFO closure with the ASOC device at our institution between July 2010 and December 2014. Demographic and clinical characteristics were obtained. All available pre- and intraprocedural echocardiography, including transthoracic echocardiography (TTE), transesophageal echocardiography (TEE), and intracardiac echocardiography (ICE) were evaluated. Preprocedure TTE studies were reviewed for the presence of atrial septal aneurysm (ASA), which was universally defined as atrial septal excursion $>15 \mathrm{~mm}$. Pre- and intra-procedural TEE and ICE were reviewed for the presence of ASA and abnormal thickening of the atrial septum (defined as $>4 \mathrm{~mm}$, which is the waist length of the ASOC device used in this study). In addition, measurements of pertinent atrial anatomy were also completed, including the presence and length of any appreciable PFO tract and fossa ovalis diameter. All cases were performed using the ASOC self-expanding, double-disc device. The device has a double-disc design and comprises Nitinol mesh and polyester fabric. It is manufactured in four disc diameter sizes: $18,25,30$, and $35 \mathrm{~mm}$. The 40- $\mathrm{mm}$ device is not currently available in the United States. Device size selection was at the discretion of the attending interventional cardiologist.

Univariate analyses of each measured variable were performed to assess for association with device size selection. Fisher's exact test was used to examine the association between the individual categorical variables and the size of the final closure device implanted. One-way analysis of variance was used for univariate analysis of continuous variables. Categorical variables that were individually associated with device size were combined with pertinent clinical variables to create a total of seven hypothesized prediction scores. These scores were tested with Kruskal-Wallis H Test and Fisher's exact test to assess the association between the proposed scoring system's score and the size of final closure device implanted. In addition, receiver operating characteristic $(\mathrm{ROC})$ curves were generated to assess the sensitivity and specificity of the scores. $\mathrm{p}<0.05$ was considered significant. All statistical analysis was performed using STATA (StataCorp, College Station, TX, USA).

\section{Results}

A total of 36 patients underwent percutaneous PFO closure during the study period. Patient demographics, pre- and intraprocedural imaging, and PFO characteristics are listed in Table 1. The indication for closure was cryptogenic stroke/transient ischemic attack in $75 \%$ of cases. Refractory hypoxemia and overwhelming deep vein thrombosis burden accounted for the remaining $25 \%$ of cases. Each case (100\%) was successful, with complete resolution of shunt at the 6-month follow-up. No cases were complicated by erosion or device embolization. In three cases, a smaller device was initially delivered across the PFO before it was determined to be too small and then removed prior to the delivery and deployment of a larger device. Two of these cases resulted in changes from 25 - to $30-\mathrm{mm}$ diameter devices. One case 
Table 1: Summary characteristics of patients studied

\begin{tabular}{ll}
\hline No. of Patients & 36 \\
Patient Characteristics & Mean (STD) \\
Age & $56.7(13.8)$ \\
Male & $13(36 \%)$ \\
Height (cm) & $168.7(11.1)$ \\
Weight (kg) & $86.3(25.5)$ \\
BSA & $1.9(.28)$ \\
Size of Device Implanted & \\
25 mm Cribriform & $18(50 \%)$ \\
30 mm Cribriform & $16(44 \%)$ \\
35 mm Cribriform & $2(6 \%)$ \\
Indication for Procedure & \\
Cryptogenic Stroke/TIA & $27(75 \%)$ \\
High DVT Burden & $3(8 \%)$ \\
Refractory Hypoxia & $6(17 \%)$ \\
TTE, Pre-Procedure, no. & $26(72 \%)$ \\
TEE, Pre-procedure, no. & $19(53 \%)$ \\
ICE, Intra-procedure, no. & $12(33 \%)$ \\
\hline BSA = Body Surface Area, calculated by DuBois criteria. \\
TIA = transient ischemic attack. \\
TTE = trans-thoracic echocardiography. \\
ICE = transesophogeal echocardiography. intra-cardiac echocardiography. \\
\hline
\end{tabular}

was a change from a 30- to a 35-mm diameter device. These cases were categorized by the final device size implanted. All procedures were performed using the ASOC device in one of three disc diameters: $25 \mathrm{~mm}$ (18 patients, 50\%), $30 \mathrm{~mm}$ (16 patients, 44\%), or $35 \mathrm{~mm}$ ( 2 patients, $6 \%$ ).

The results of univariate analysis are listed in Table 2. The presence of ASA ( $p=0.027$, Fisher's exact test) and PFO tunnel length $>10 \mathrm{~mm}(p=0.038$, Fisher's exact test) were independently associated with increased device size.

To guide sizing, a list of hypothesized sizing scores and their included variables is available in Table 3. A scoring system of 1 point for male sex, 1 point for ASA, and 1 point for PFO tunnel $>10 \mathrm{~mm}$ in length was significantly associated with the size of closure device implanted ( $p=0.007$ and $p=0.006$, KruskalWallis $\mathrm{H}$ Test, STATA; Figure 1). ROC curves were created for each prediction score to assess sensitivity and specificity. Because of the limited number of $35-\mathrm{mm}$ devices implanted in the study period $(n=2)$, 30- and 35-mm devices were grouped together for the ROC analysis. The area under the curve for the proposed sizing score was 0.8846 (STATA, Figure 2). The cutpoint score of $\geq 2$ had $75 \%$ sensitivity and $92 \%$ specificity for a device diameter $\geq 30 \mathrm{~mm}$.

\section{Discussion}

Percutaneous PFO closure has been proposed as a safe alternative or adjunct therapy to antiplatelet medication and anticoagulation in patients with cryptogenic stroke or peripheral embolism [10, 17-19]. Observational studies have suggested a substantial benefit to PFO closure in the secondary prevention of neurologic and vascular events when compared to medical therapy $[20,21]$. Three randomized controlled trials assessing PFO closure for secondary prevention of cryptogenic stroke failed to reach their primary efficacy endpoints [22-24]. However, per-protocol and as-treated analysis of one of the randomized trials did show a significant benefit of closure over medical therapy in the prevention of recurrent ischemic stroke and death [22]. At this point, percutaneous closure of PFOs in the U.S. is considered "off-label," and there is a wide heterogeneity in device size selection.

Our study suggests that patients undergoing percutaneous PFO closure who display certain echocardiographic findings will likely require closure devices with larger disc diameters. The presence of ASA and increasing length of PFO tunnel on TEE or ICE were independently associated with larger device size. In addition, our findings suggest that a sizing score may be used to facilitate identification of patients that are likely to require larger devices. A scoring system consisting of 1 point for the presence of ASA, 1 point for PFO tunnel length $>10 \mathrm{~mm}$, and 1 point for male sex was statistically associated with increasing device size. Scores of 0 and 1 were associated with the $25 \mathrm{~mm}$ device. A score of 2 was associated with the $30 \mathrm{~mm}$ device, and a score of 3 was associated with the $35 \mathrm{~mm}$ device. 
Table 2: Univariate Analysis of Patient Characteristics and Echocardiographic Findings

\begin{tabular}{|c|c|c|c|c|}
\hline & $25 \mathrm{~mm}$ Device & $30 \mathrm{~mm}$ Device & $35 \mathrm{~mm}$ Device & \\
\hline \multicolumn{5}{|l|}{ Variable } \\
\hline No. of patients & 18 & 16 & 2 & \\
\hline No. Male & 7 (39\%) & $5(31 \%)$ & $1(50 \%)$ & $p=0.87$ \\
\hline Age & $57(12.5)$ & $55(16.2)$ & $61.5(4.9)$ & $p=0.82$ \\
\hline Height (cm) & $170(12)$ & $166(9)$ & $176(16)$ & $p=0.43$ \\
\hline Weight (kg) & $89.7(31)$ & $83.7(19)$ & $76(8)$ & $p=0.68$ \\
\hline BSA & $2.0(0.3)$ & $1.9(0.2)$ & $1.95(0.2)$ & $p=0.75$ \\
\hline Tunnel Length (mm) & $8.7(3.2)$ & $14.2(6.5)$ & 22 & $p=0.007$ \\
\hline \multicolumn{5}{|l|}{ Percent with: } \\
\hline ASA & $3(17 \%)$ & $9(56 \%)$ & $1(50 \%)$ & $p=0.027$ \\
\hline Long Tunnel & $4(31 \%)$ & $6(86 \%)$ & $1(100 \%)$ & $p=0.038$ \\
\hline Thickened Septum & 7 (39\%) & $5(31 \%)$ & $1(50 \%)$ & $p=0.871$ \\
\hline
\end{tabular}

Table 3: List of hypothesized sizing scores tested

\begin{tabular}{lll}
\hline Score & Variables Included in Score \\
\hline A & $p=0.26$ & 1 point each for male sex and presence of ASA \\
B & $p=0.08$ & 1 point each for male sex, presence of ASA, and thickened septum \\
C & $p=0.41$ & 1 point each for male sex, tall height, and presence of ASA \\
D & $p=0.29$ & 1 point each for presence of ASA and thickened septum \\
E & $p=0.21$ & 1 point each for thickened septum, tall height, and presence of ASA \\
F & $p=0.03$ & 1 point each for male sex, presence of ASA, thickened septum, and long tunnel \\
G & $p=0.007$ & 1 point each for male sex, presence of ASA, and long tunnel \\
\hline
\end{tabular}

ASA = Atrial Septal Aneurysm, defined as atrial septal excursion of $>15 \mathrm{~mm}$. Long Tunnel $=$ PFO Tunnel Length on TEE or ICE $>10 \mathrm{~mm}$ in length.

$P$ values listed are results of Fisher's Exact Test using STATA.

At this time there is no consensus regarding the selection of device size for PFO closure. A method utilizing a sizing balloon to estimate appropriate device size has been described previously [15]. However, the use of sizing balloons carries some risk and may not provide meaningful information due to the relative compliance of the tissues of the intra-atrial septum [16]. A sizing balloon sitting across a slit-like or ovalshaped PFO may not adequately measure defect length; therefore, operators typically select the size of closure device based on personal experience. This practice was reflected in the clinical trials assessing percutaneous PFO closure. In the RESPECT trial, the choice of device size was made at the discretion of the operator with the guidance of the Amplatzer PFO Occluder instructions for use [22]. This device has a larger right atrial (RA) disc than left atrial (LA) disc with a central pin. Specifically, the instructions suggest measur- 


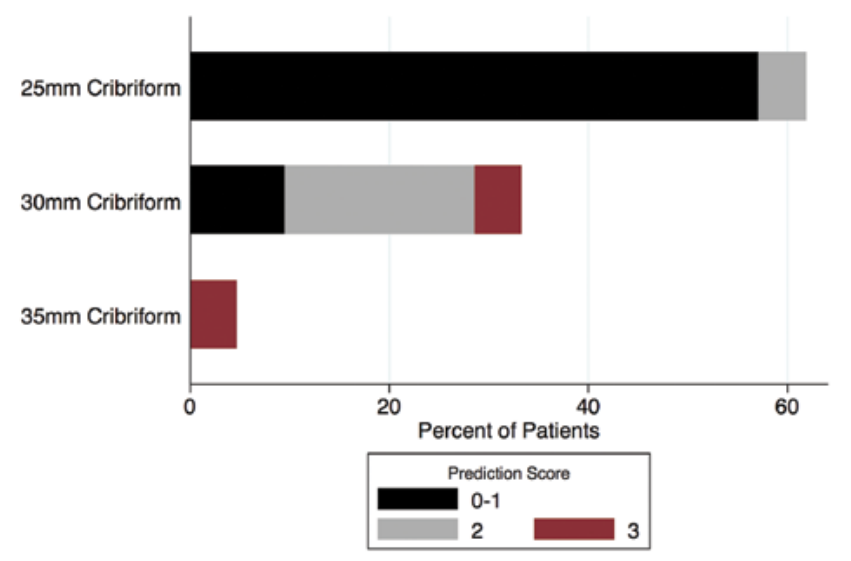

Figure 1. Percent of patients in study receiving each device size and their sizing score.The score includes 1 point for male sex, 1 point for an atrial septal aneurysm (septal excursion $>15 \mathrm{~mm}$ on echocardiography), and 1 point for a patent foramen ovale tunnel $>10 \mathrm{~mm}$ in length.

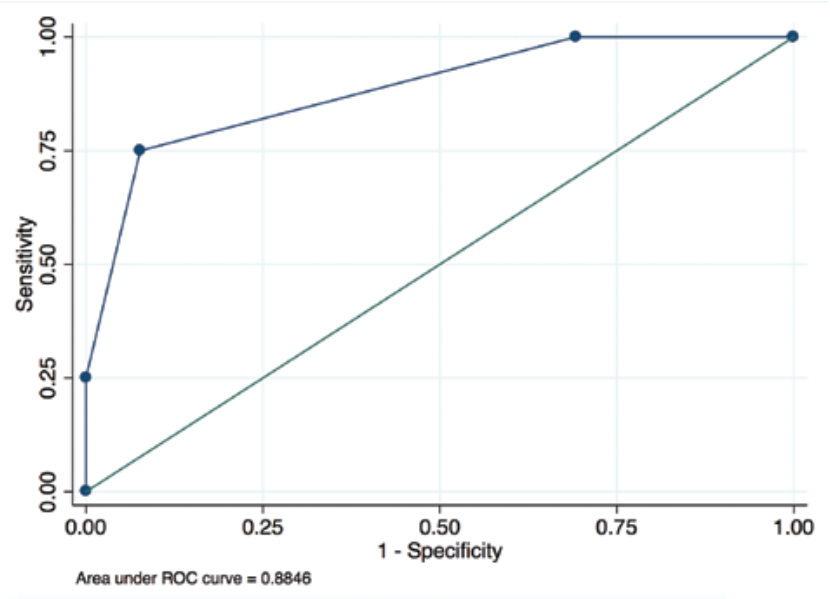

Figure 2. Receiver operating characteristic curve of the sizing score for association with implantation of an Amplatzer Septal Occluder "Cribriform" device $\geq 30 \mathrm{~mm}$ diameter. The score includes 1 point for male sex, 1 point for an atrial septal aneurysm (septal excursion $>15 \mathrm{~mm}$ on echocardiography), and 1 point for a patent foramen ovale tunnel $>10 \mathrm{~mm}$ in length.

ing the shortest distance from the PFO to the aortic root and SVC. The recommendations call for implantation of the $35 \mathrm{~mm}$ device if this distance is $>17.5 \mathrm{~mm}$, the $25 \mathrm{~mm}$ device if the distance is $12.5-17.4 \mathrm{~mm}$, and a 1-8 $\mathrm{mm}$ device if the distance is $9-12.4 \mathrm{~mm}$. If the shortest distance from the PFO to the aortic root or
SVC is $<9 \mathrm{~mm}$, the instructions prohibit implanting the Amplatzer PFO Occluder device. The instructions do allow for the use of a larger device in the setting of ASA, but there are not specific recommendations for device size selection when ASA is present. These recommendations rely on obtaining echocardiographic measurements from the PFO to both the aortic root and SVC. These measurements may be difficult to reproduce, especially in mobile, aneurysmal atrial septa. In addition, the instructions do not account for BMI or tunnel length, which could contribute to device sliding if smaller devices are chosen. It should be noted that the Amplatzer PFO Occluder has a larger RA disc than LA disc, for example, the $35 \mathrm{~mm}$ device has a 25 $\mathrm{mm}$ left atrial disc. The difference in disc size may also contribute to device sizing, especially compared to the ASOC device that has discs of equal sizes. The CLOSURE 1 trial protocol called for sizing balloon estimation of PFO size prior to closure but did not delineate how this would inform device size selection [24]. Balloon-sizing was optional in the PC trial, and device size selection was at the discretion of the treating physician [23].

This study identified two echocardiographic findings that were independently associated with larger device requirements for PFO closure: the presence of ASA and a long PFO tunnel. Aneurysm of the interatrial septum is characterized by mobile atrial tissue that has a large distance of excursion during the cardiac cycle. This floppy tissue provides very little support for a closure device deployed across a PFO. Therefore, a larger device may be beneficial in such cases to provide additional support of septal architecture and continuously occlude the PFO. Long-tunnel PFO tracts are challenging for device closure because they require devices with either variable waist-length or large, strong discs to effectively shorten the tunnel length. All of the closures completed in our study were performed with the ASOC device, which is a strong double-disc device. Larger disc diameters of the ASOC device could provide additional strength to effectively shorten the long PFO tunnel length, which may explain why larger disc devices were used in the patients with longer tunnels. Interestingly, both ASA and long-tunnel length have been associated with risk for embolization, which may indicate that patients with these findings would benefit from larger 
devices to avoid embolization [13]. There was no correlation between the presence of a right atrial Chiari network and device size selection.

Objective selection of device size with the proposed scoring system could potentially improve both the safety and cost of percutaneous PFO closure. For example, three patients in our cohort required extended procedure times to remove a device that was too small before implanting an appropriately sized device. Two of these patients had ASA on preprocedure TTE, and were, therefore, more likely to require a larger device size. Prediction of device size requirements with the sizing score could have limited fluoroscopy time and prevented the waste of a device that was too small.

\section{Limitations}

This was single-center, retrospective review of 36 patients who underwent percutaneous PFO closure with the ASOC device. No other devices were studied. Alternative devices may need additional assessment for sizing. Only 21 patients had either TEE or ICE images available for retrospective review, further limiting the functional sample size of the study. Only two patients received the largest device $(35 \mathrm{~mm})$, which limits any conclusions regarding association with this device size. The choice of closure device size was made at the discretion of the interventional cardiologist; therefore, our findings may be confounded by any subjective bias the operator applied in selecting a closure device. In addition, the retrospective nature of this study did not allow us to adequately assess whether the device size truly represents the optimal-sized device for each patient. Larger, prospective studies are needed to clarify this point.

\section{Conclusions}

The presence of ASA and a PFO tunnel length $>10 \mathrm{~mm}$ were independently associated with the selection of a larger PFO closure device in this cohort of patients. A scoring system consisting of 1 point for the presence of ASA, 1 point for PFO tunnel length $>10 \mathrm{~mm}$, and 1 point for male sex was statistically associated with the size of device implanted. Using the scoring thresholds defined above, this score could be implemented to predict the appropriate device size required in a specific patient. Use of this prediction score could both improve patient safety and limit costs. Further prospective studies are warranted to determine the accuracy of this sizing score and whether this its use limits residual shunt and other adverse events related to percutaneous PFO closure.

\section{Conflict of Interest}

Dr. Shah is a consultant/proctor for St. Jude Medical. None of the other authors have conflicts of interest to report.

\section{Comment on this Article or Ask a Question}

\section{References}

1. Hagen PT, Scholz DG, Edwards WD. Incidence and size of patent foramen ovale during the first 10 decades of life: an autopsy study of 965 normal hearts. Mayo Clin Proc. 1984;59:17-20. DOI: 10.1016/ S0025-6196(12)60336-X

2. Meissner I, Whisnant JP, Khandheria BK, Spittell PC, O'Fallon WM, Pascoe RD, et al. Prevalence of potential risk factors for stroke assessed by transesophageal echocardiography and carotid ultrasonography: the SPARC study. Stroke Prevention: assessment of Risk in a Community. Mayo Clin Proc. 1999;74:862-869. DOI: $10.4065 / 74.9 .862$
3. Lechat $P$, Mas $J$, Lascault $G$, Loron $P$, Theard M, Klimczac $M$, et al. Prevalence of patent foramen ovale in patients with stroke. N Engl J Med. 1988;318:1148-1152. DOI: 10.1056/NEJM198805053181802

4. Webster MW, Chancellor AM, Smith HJ, Swift DL, Sharpe DN, Bass NM, et al. Patent foramen ovale in young stroke patients. Lancet. 1988;332:11-12. DOI: 10.1016/ S0140-6736(88)92944-3

5. Hausmann D, Mügge A, Daniel WG. Identification of patent foramen ovale permitting paradoxic embolism. J Am Coll Cardiol. 1995;26:1030-1038. DOI: 10.1016/07351097(95)00288-9
6. Wöhrle J, Kochs M, Hombach V, Merkle $\mathrm{N}$. Prevalence of myocardial scar in patients with cryptogenic cerebral ischemic events and patent foramen ovale. JACC Cardiovasc Imaging. 2010;3:833-839. DOI: 10.1016/j.jcmg.2010.05.013

7. Bonati LH, Kessel-Schaefer A, Linka AZ, Buser P, Wetzel SG, Radue EW, et al. Diffusion-weighted imaging in stroke attributable to patent foramen ovale: significance of concomitant atrial septum aneurysm. Stroke. 2006;37:2030-2034. DOI: 10.1161/01.STR.0000231655.52686.ab

8. Kerut EK, Norfleet WT, Plotnick GD, Giles TD. Patent foramen ovale: a review of 
associated conditions and the impact of physiological size. J Am Coll Cardiol. 2001;38:613-623. DOI: 10.1016/S07351097(01)01427-9

9. Wahl A, Praz F, Tai T, Findling O, Walpoth $\mathrm{N}$, Nedeltchev K, et al. Improvement of migraine headaches after percutaneous closure of patent foramen ovale for secondary prevention of paradoxical embolism. Heart. 2010;96:967-973. DOI: 10.1136/ hrt.2009.181156

10. Godart F, Rey C, Prat A, Vincentelli A, Chmaït $A$, Francart $C$, et al. Atrial right-to-left shunting causing severe hypoxaemia despite normal right-sided pressures. Report of 11 consecutive cases corrected by percutaneous closure. Eur Heart J. 2000;21:483-489. DOI: 10.1053/euhj.1999.1944

11. Amin Z. Echocardiographic predictors of cardiac erosion after amplatzer septal occluder placement. Catheter Cardiovasc Interv. 2014;83:84-92. DOI: 10.1002/ ccd. 25175

12. Wagdi P. Incidence and predictors of atrial fibrillation following transcatheter closure of interatrial septal communications using contemporary devices. Clin Res Cardiol. 2010;99:507-510. DOI: 10.1007/s00392010-0149-3

13. Goel SS, Olcay A, Tuzcu EM, Krasuski RA, Kapadia SR. Embolization of patent foramen ovale closure devices: incidence, role of imaging in identification, potential causes, and management. Texas Hear Inst J. 2013;40:439444. DOI: 10.1016/j.jcin.2011.01.009

14. AGA Medical Corporation. Multifenestrated Septal Occluder - "Cribriform." Instructions for Use. St. Paul: AGA Medical Corporation. 2011:1-10.

15. Alibegovic J, Bonvini R, Sigwart U, Dorsaz P,
Camenzind $\mathrm{E}$, Verin V. The role of the sizing balloon in selection of the patent foramen ovale closure device size. Exp Clin Cardiol. 2008;13:42-46. PMID: 18650972

16. Bonvini RF, Sigwart U, Verin V. Interatrial septum rupture during balloon measurement of a patent foramen ovale in a young patient presenting cryptogenic stroke. Catheter Cardiovasc Interv. 2007;69:274276. DOI: $10.1002 / \mathrm{ccd} .20969$

17. Inglessis I, Elmariah $S$, Rengifo-Moreno PA, Margey R, O'Callaghan C, Cruz-Gonzalez $\mathrm{I}$, et al. Long-term experience and outcomes with transcatheter closure of patent foramen ovale. JACC Cardiovasc Interv. 2013;6:1176-1183. DOI: 10.1016/j. jcin.2013.06.013

18. Bissessor N, Wong AW, Hourigan LA, Jayasinghe RS, Scalia GS, Burstow DJ, et al. Percutaneous patent foramen ovale closure: outcomes with the Premere and Amplatzer devices. Cardiovasc Revasc Med. 2011;12:164-169. DOI: 10.1016/j.carrev.2010.06.001

19. Wahl A, Tai T, Praz F, Schwerzmann M, Seiler $C$, Nedeltchev $K$, et al. Late results after percutaneous closure of patent foramen ovale for secondary prevention of paradoxical embolism using the amplatzer PFO occluder without intraprocedural echocardiography: effect of device size. JACC Cardiovasc Interv. 2009;2:116-123. DOI: 10.1016/j.jcin.2008.09.013

20. Wahl $A$, Jüni $P$, Mono $M L$, Kalesan $B$, Praz F, Geister L, et al. Long-term propensity score-matched comparison of percutaneous closure of patent foramen ovale with medical treatment after paradoxical embolism. Circulation. 2012;125:803-812. DOI: 10.1161/CIRCU-
LATIONAHA.111.030494

21. Khan AR, Bin Abdulhak AA, Sheikh MA, Khan S, Erwin PJ, Tleyheh I, et al. Device closure of patent foramen ovale versus medical therapy in cryptogenic stroke: a systematic review and meta-analysis. JACC Cardiovasc Interv. 2013;6:1316-1323. DOI: 10.1016/j.jcin.2013.08.001

22. Carroll JD, Saver JL, Thaler DE, Smalling RW, Berry S, MacDonalad LA, et al. Closure of patent foramen ovale versus medical therapy after cryptogenic stroke. $\mathrm{N}$ Engl J Med. 2013;368:1092-1100. DOI: 10.1056/ NEJMoa1301440

23. Meier B, Kalesan B, Mattle HP, Khattab AA, Hildick-Smith $D$, Dudek $D$, et al. Percutaneous closure of patent foramen ovale in cryptogenic embolism. N Engl J Med. 2013;368:1083-1091. DOI: 10.1056/NEJMoa1211716

24. Furlan AJ, Reisman M, Massaro J, Mauri L, Adams H, Albers GW, et al. Closure or medical therapy for cryptogenic stroke with patent foramen ovale. N Engl J Med. 2012;366:991-999. DOI: 10.1056/NEJMoa1009639

Cite this article as: Venturini JM, Retzer EM, Estrada JR, Mediratta A, Friant J, Nathan S, Paul JD, Blair J, Lang RM, Shah AP. A Practical Scoring System to Select Optimally Sized Devices for Percutaneous Patent Foramen Ovale Closure. Journal of Structural Heart Disease. 2016;2(5):217-223. DOl: http:// dx.doi.org/10.12945/j.jshd.2016.009.15 